\title{
Sports-related head injury as a cause of ossicular trauma
}

\begin{abstract}
Background: Ossicular trauma can come from many sources; however, the general cause of ossicular trauma results from temporal bone injury. Temporal bone injury is commonly classified as a concussion, or head injury. Causes for head injury are predominantly from car accidents, but the remaining causes can range from a fall to a gunshot wound. Incidentally, another cause for head injuries, particularly temporal bone injuries, is sports-related traumas.

Discussion: Sports-related injuries can cause serious temporal damage than affect ossicular function. Potential damage of the ossicles includes ossicular discontinuity, disarticulation, or extrusion. Some ossicular trauma may be secondary to another middle ear cavity trauma. While temporal bone injuries can really impact any part of the auditory and balance systems, the discussion of these articles focuses on how sports-related head injuries can affect the function of the ossicular chain.
\end{abstract}

Conclusion: Damage to the ossicular chain may seem like an unlikely injury; however the lack of a full support system makes the ossicles inclined to damage, especially with any blunt force traumas. Sports can be an unfortunate cause for head injuries and temporal bone injuries, which can impact the structure and function of the ossicular chain.

Keywords: ossicular trauma, ossicular chain, sports injury, temporal bone injury
Volume 10 Issue 5 - 2018

\author{
Lilian Felipe \\ Department of Speech and Hearing Sciences, Assistant \\ Professor, Lamar University, USA
}

\begin{abstract}
Correspondence: Lilian Felipe, Department of Speech and Hearing Sciences, Assistant Professor, Lamar University, P.O. Box 10076, Beaumont, TX 777I0, USA, Tel (409) 880 7900, Email lfelape@lamar.edu
\end{abstract}

Received: October 04, 2018 | Published: October 24, 2018

\section{Introduction}

The middle ear is the part of the ear between the tympanic membrane and the oval window, and consists of three bones: the malleus, the incus and the stapes. The purpose of the auditory ossicles (or ossicular chain) is to transmit sound via a chain reaction of vibrations that connects the eardrum to the inner ear and cochlea. The auditory chain reaction starts when sound reaches the tympanic membrane. The vibrational pressure is passed to the malleus, an articulated bone that flexes at one of the two incudomalleolar joints. The vibration is then passed to the incus which flexes at another incudomalleolar joint before transferring the impulses to the stapes. The role of auditory ossicles is complete when the vibrational input is passed to the cochlea through the oval window. The auditory ossicles serve an important role in hearing in that they transition sound waves from the air to the fluid-filled core the cochlea. They effectively amp up the vibrations by around 30 decibels $(\mathrm{dB})$ to accommodate this transition.

Damage to the middle ear can occur in various ways, with the most common source being otitis media. Dysfunction of the eustachian tube becomes the prime contributor for otitis media as the eustachian tube swells and fails to move air out of the middle ear cavity. ${ }^{1}$ As pressure is not equalized within the middle ear cavity, it can be easy for fluid and bacteria to accumulate and begin to affects surrounding structures, such as the tympanic membrane and the ossicles (middle ear bones). While damage through an infection or damage to the tympanic membrane is one of the first thoughts for damage to the auditory system, it seems to be overlooked that damage can easily afflict the ossicles of the middle ear. ${ }^{1,2}$

It is important to understand that although these are three bones within the middle ear cavity, these bones are miniscule in comparison to other bones of the body. The auditory ossicles are a chain of small bones in the middle ear that transmit sound from the outer ear to the inner ear through mechanical vibration. Additionally, these bones are fixed to each other will little to no surrounding support in some areas; it can appear as if they are floating. Therefore, the lack of support makes is easy to dislocate the ossicular chain. ${ }^{2}$ While general damage to the ossicular chain can be congenital or from infections, dislocation of the ossicular chain mainly occurs from trauma, particularly head injuries of the temporal lobe. ${ }^{2}$ The temporal bone houses much of the auditory system, particularly the middle ear cavity. This means that any blunt trauma to the side of the head, where the temporal bone is located, runs the risk of bringing dislocation to the ossicles. Specifically, the trauma to the temporal bone can be instantaneously disruptive and weakens the ligaments that stabilize and connect the ossicular chain. ${ }^{3}$ The most common cause of head trauma is due to traffic accidents, but another important cause to be aware of, and that is on the rise, is due to sports. ${ }^{4}$ The intense physical contact of some sports, such as but not exclusive to football and diving, can cause harsh impacts and particularly to the head. While helmets used for various sports can dramatically minimize the damage to the skull and surrounding structures, helmets cannot fully prevent forcefully impactful blows to a person's head. ${ }^{5}$ This article specifically focused on sports and ossicular trauma, and how head traumas from sports can negatively impact the ossicular chain of the auditory system.

\section{Discussion}

Head injuries by cause of sports are prevalent, particularly in children and adolescents, accounting for approximately $21 \%$ of head injuries. ${ }^{6}$ In sports, it is reflexive for athletes, and people in general, to turn their head to the side in order to take the impact of the trauma coming towards their head. ${ }^{5}$ 
It is logical that one would instinctually and reflexively turn their head to avoid direct/frontal damage to the head and face. Assuming this is a reflex for imminent trauma in a sport, then the impact of a blow can be quite damaging to the temporal bone and ultimately the ossicular chain of the auditory system. A damage to the ossicular chain is called ossicular chain dislocation and is also referred to as ossicular discontinuity or ossicular disarticulation. ${ }^{1,2,4,7,8}$

The typical locations for ossicular dislocation are, primarily, the incudostapedial joint and, rarely, the incudomalleolar joint. ${ }^{3,8}$ While it is easy to think simplify the damage to the ossicular chain as simply a dislocation or a subluxation, fractures to the ossicles can also occur due the sustained head trauma from the sport. ${ }^{1,2,4,5,7,8}$ The typical location for ossicular fractures are at the stapes crura and the incus., ${ }^{3,7}$ However, fractures of the ossicles are uncommon in comparison to dislocation, but fractures are possible due to the intensity of the blunt sport trauma. ${ }^{2}$ The third kind of damage that can occur to the ossicular chain, and the least common, is extrusion of the ossicle due to head trauma. ${ }^{1,2,3}$ This extrusion can occur either through the tympanic membrane and into the external auditory canal or into the inner ear, but these occurrences are quite rare.

Some noticeable symptoms of ossicular chain dislocation include hypercompliant tympanograms, reduced or absent acoustic reflexes, and a conductive hearing loss. The consensus for treatment of damage to the ossicular chain is surgical intervention, called an ossiculoplasty. ${ }^{1,2,3,4,5,7,8}$ In this surgery, the surgeon will attempt to realign or mend the affected ossicles. If other procedures are needed, like a tympanoplasty or mastoidectomy, then this will also occur during the surgery.

\section{Conclusion}

Damage to the middle ear, and the entire auditory system, can occur in many ways. The more common ways are infection and congenital abnormalities; however, traumas to the temporal bone can cause serious damage to the system as well. While traffic accidents are the most common cause for head injury, sports are also a main contributor. Future research should aim to discuss this topic more extensively and to discuss identification, safety, and prevention.

\section{Acknowledgments}

None.

\section{Conflict of interest}

The author declares there is no conflict of interest

\section{References}

1. Musiek FE, Baran JA, Shinn JB, et al. Disorders of the auditory system Plural Publishing. 2011:978-1-59756-350-355.

2. Gupta M, Singh S, Gupta M. Traumatic ossicle extrusion into the external auditory canal. Ear Nose Throat J. 2013:92(6):E21.

3. Kinney SE. Trauma to the middle ear and temporal bone. Otolaryngology Head Neck Surgery. St Louis: Mosby, 1998: pp. 3076-3087.

4. Khatri DK, Rao MI, Ali M. Pattern of injuries regarding ear, nose, throat $\&$ head \& neck in patients presenting in accident \& emergency. Pakistan Journal of Surgery. 2016;32(4):255-259.

5. Wagner GA. Sports Injuries of the Ear. Canadian Family Physician. 1972;18(7):51-53.

6. American Association of Neurological Surgeons (AANS). Sports-related head injury. AANS Publication. 2018.

7. Park GY, Choi JE, Cho YS. Traumatic ossicular disruption with isolated fracture of the stapes suprastructure: Comparison with incudostapedial joint dislocation. Acta Oto-laryngologica. 2014;134(12):1225-1230.

8. Sasindran V, Joseph A, Babu B, et al. A case of posttraumatic incudomalleolar disruption. Indian Journal of Otology. 2014;20(2):89-91. 